\title{
A Rigorous Method for Compensation Selection and Alignment of Microlithographic Optical Systems
}

\author{
H. N. Chapman \\ D. W. Sweeney
}

This paper was prepared for and presented to the 23rd Annual International Symposium on Microlithography Santa Clara, California

February 22-27, 1998

March 25, 1998

This is a preprint of a paper intended for publication in a journal or proceedings. Since changes may be made before publication, this preprint is made available with the understanding that it will not be cited or reproduced without the permission of the author. 


\section{DISCLAIMER}

This document was prepared as an account of work sponsored by an agency of the United States Government. Neither the United States Government nor the University of California nor any of their employees, makes any warranty, express or implied, or assumes any legal liability or responsibility for the accuracy, completeness, or usefulness of any information, apparatus, product, or process

disclosed, or represents that its use would not infringe privately owned rights. Reference herein to any specific commercial product, process, or service by trade name, trademark, manufacturer, or otherwise, does not necessarily constitute or imply its endorsement, recommendation, or favoring by the United States Government or the University of California. The views and opinions of authors expressed herein do not necessarily state or reflect those of the United States Government or the University of California, and shall not be used for advertising or product endorsement purposes. 


\title{
A Rigorous Method for Compensation Selection and Alignment of Microlithographic Optical Systems
}

\author{
Henry N. Chapman and Donald W. Sweeney \\ Lawrence Livermore National Laboratory, Livermore, CA 94551, USA.
}

\begin{abstract}
The assembly of an optical system requires the correction of aberrations in the entire imaging field by making selected rigid-body motions of the optical elements. We present a rigorous method for determining which adjustment motions, called compensators, to use for alignment. These compensators are found by employing techniques from linear algebra that choose the most independent vectors from a set which are interdependent. The method finds the smallest number of compensators to correct for misalignments of a given magnitude. As an example the method is applied to a four-mirror scanning ring-field EUV lithography system. It is shown that out of 32 degrees of freedom in the configuration of the optical elements, only eight compensators are required on the optics. By adjusting these compensators a misaligned configuration giving $30 \lambda$ wavefront error can be assembled to $\lambda / 50$ in the absense of measurement noise.
\end{abstract}

Keywords: Alignment, assembly, tolerancing, EUV projection lithography, SVD, compensator

\section{INTRODUCTION}

A four-mirror ring-field EUV lithography system is being developed to operate at a wavelength of $13.4 \mathrm{~nm}$ and print patterns with critical dimensions below $0.1 \mu \mathrm{m}$ in a large scanned field. Assembling this system will be an extremely challenging task. All but one of the mirrors will be aspheres that are used off axis. The full aspheres will not be manufactured, nor will there be any optical reference to determine the relationship between a mirror and the optic axis. Thus, as far as assembly is concerned, the camera can be thought of as a decentered optical system. Projection optics for EUV lithography must be assembled to give wavefront errors no greater than about $0.02 \lambda$ RMS,1] corresponding to $0.27 \mathrm{~nm}$ RMS. The prototype four-mirror ring-field system currently under development is predicted to have a more modest residual wavefront error of $0.07 \lambda \mathrm{RMS}$, due to figure specification of the optics.2] However, alignment can be performed, to the extrordinary tolerances required of production instruments, using new interferometers that have recently been developed. These operate at visible $e^{3}$ and EUV ${ }^{4}$ wavelengths and can measure to accuracies better than $0.25 \mathrm{~nm}$ RMS and $0.05 \mathrm{~nm}$ RMS, respectively.

A common method to assemble precision optical systems is to use interferograms that are measured at several field points $\frac{516}{5}$ The wavefront measurements are used to determine what corrections need to be made to the positions of the optics. In the assembly of microlithographic lenses, individual aberrations are usually corrected in turn by making adjustments of predetermined compensators ${ }^{7}$ This can be done because there are usually a large number of elements and the surfaces are spherical. In the case of the EUV projection optics, however, there are complex interrelationships between the adjustments and simple corrections can not be found for each aberration. The successful alignment of the EUV projection optics requires carefully selecting which adjustments to use as compensators and knowing what measurements are sufficient to determine the misaligned state of the system.

In the assembly and operation of the optical system one would wish to use as few compensators as necessary, so that the optical mounts can be optimized for stiffness and stability. In this paper we present a method, based on results from linear algebra, to determine the best set of compensators to correct for aberrations induced by misalignment. The best set of compensators is the one that addresses the system aberration field in the most orthogonal, or independent, way. The number and choice of compensators depends on how large a misalignment one wishes to correct, and the relationship between the number of compensators and the magnitude of correctable misalignments is investigated.

The method of alignment described in this paper is an iterative Newton-type method,, 8 utilising the singular value decomposition (SVD). This is very similar to Grey's orthogonalisation method used in optical design,, 9 is preferred since it overcomes the ill-posed problem of alignment by regulating the magnitude of the correction. 


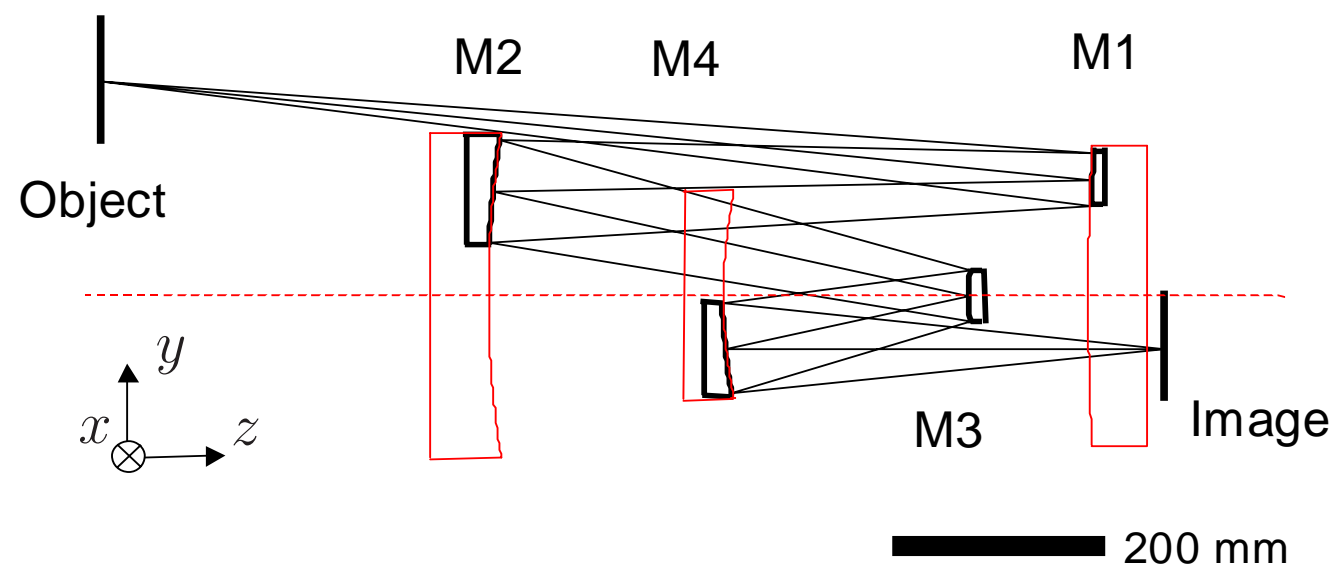

Figure 1. Schematic diagram of the EUV lithographic camera, showing the mirrors that will be constructed (bold outlines) as well as the rotationally symmetric parents from which they were derived (thin outlines). The well-corrected field is an arc of an annulus centered on the optic axis. Rays are shown for one field point.

The convergence of the method is quadratic ${ }^{8}$ which means that alignment can be achieved with a small number of measurement steps. We investigate the alignment method by performing a large number of simulations, and find that the method is robust to measurement noise and imperfect optical surfaces. The simulations confirm the analysis of compensator selection and the magnitude of correctable misalignments.

\section{THE EUV PROJECTION OPTICS}

The EUV lithographic camera is a four-mirror ring-field design which operates at a wavelength of $13.4 \mathrm{~nm}$ and a demagnification of $4 \times ! 10$ The used field is a $30^{\circ}$ arc of the ring-field with a width at the object plane of only $6 \mathrm{~mm}$ compared with the chord length of $104 \mathrm{~mm}$. Images will be printed by synchronously scanning the wafer at a quarter of the speed of the mask, so that the moving image will appear stationary on the wafer. A schematic of the optical design is shown in Fig. 1 Three of the four mirrors are aspheric, with the stop located on a spherical surface (mirror M3). The design is rotationally symmetric about the optic axis but, since the used field is an arc of an annulus located far from the axis, the clear aperture of each of the aspheres consists of an off-axis region of the full parent. Only a region about the clear aperture will actually be manufactured.

Each of the optical surfaces has five rigid-body degrees of freedom. We define the configuration of the optical system in terms of translations and rotations of the optics. These are: $\theta_{x}$ and $\theta_{y}$, rotations around axes that are respectively parallel to the $x$ and $y$ axes (see Fig. 11) and that intersect at the center of the mirrors' clear apertures; and translations $\Delta x, \Delta y$, and $\Delta z$ along each of the $x, y$, or $z$ axes. The center of the clear aperture is defined as the point of intersection of the surface with the chief ray that originates from the central field point. Since the parents of the mirrors are rotationally symmetric, any rotation $\theta_{z}$ is equivalent to $\Delta x$ and $\Delta y$ translations, as long as the edge of the part is not rotated into the clear aperture. Although mirror M3 is spherical, and hence can be fully specified by three parameters, this mirror also contains the circular stop which requires another two parameters. The configuration of the system is completely described by the values of these parameters, which can be listed together to form the configuration vector, $\mathbf{x}$. The definition of this vector is given in Table 1 . The units of adjustment of each parameter are also listed in the table. The unit of angle was chosen as $10 \mathrm{mrad}$ by dividing the unit of length $(1 \mathrm{~mm})$ by the mean diameter of the mirror substrates (on the order of $100 \mathrm{~mm}$ ). This is so that an actuator which induces a rotation by tilting one side of an optic would have approximately the same unit length as one causing a translation of that optic.

In addition to the rigid-body degrees of freedom we include the base-sphere curvatures of the four mirrors as parameters of the configuration of the system. These of course will never be used as compensators but are included so that the compensator selection algorithm will choose rigid-body parameters that can correct for errors in the basesphere curvature. The base-sphere curvatures are expressed in units of $10^{-7} \mathrm{~mm}^{-1}$, chosen to give approximately the same magnitude of aberration as a unit change of a $z$ translation. 


\begin{tabular}{|c|c|c|c|c|}
\hline $\begin{array}{c}\text { element, } \\
j\end{array}$ & $\begin{array}{c}\text { Configuration } \\
\text { parameter }\end{array}$ & $\begin{array}{c}\text { Units of } \\
\text { adjustment }\end{array}$ & $\begin{array}{c}\text { Full error } \\
\text { capture range }\end{array}$ & $\begin{array}{c}\text { Weighting } \\
\text { for simulations }\end{array}$ \\
\hline$\overline{1}$ & $\overline{\overline{\mathrm{Ob}}-\theta_{x}}$ & $50 \mathrm{mrad}$ & $86.1 \mathrm{mrad}$ & $\overline{0.001}$ \\
\hline 2 & Ob- $\theta_{y}$ & $50 \mathrm{mrad}$ & $70.8 \mathrm{mrad}$ & 0.001 \\
\hline 3 & Ob- $\Delta x$ & $5 \mathrm{~mm}$ & $5.13 \mathrm{~mm}$ & 0.044 \\
\hline 4 & Ob- $\Delta y$ & $5 \mathrm{~mm}$ & $4.02 \mathrm{~mm}$ & 0.044 \\
\hline 5 & Ob- $\Delta z$ & $2.5 \mathrm{~mm}$ & $2.00 \mathrm{~mm}$ & 0.063 \\
\hline 6 & M1- $\theta_{x}$ & $10 \mathrm{mrad}$ & $1.14 \mathrm{mrad}$ & 0.141 \\
\hline 7 & M1- $\theta_{y}$ & $10 \mathrm{mrad}$ & $1.20 \mathrm{mrad}$ & 0.141 \\
\hline 8 & M1- $\Delta x$ & $1 \mathrm{~mm}$ & $0.35 \mathrm{~mm}$ & 0.281 \\
\hline 9 & M1- $\Delta y$ & $1 \mathrm{~mm}$ & $0.68 \mathrm{~mm}$ & 0.199 \\
\hline 10 & $\mathrm{M} 1-\Delta z$ & $0.5 \mathrm{~mm}$ & $0.69 \mathrm{~mm}$ & 0.141 \\
\hline 11 & $\mathrm{M} 2-\theta_{x}$ & $10 \mathrm{mrad}$ & $1.98 \mathrm{mrad}$ & 0.141 \\
\hline 12 & $\mathrm{M} 2-\theta_{y}$ & $10 \mathrm{mrad}$ & $1.99 \mathrm{mrad}$ & 0.141 \\
\hline 13 & M2- $\Delta x$ & $1 \mathrm{~mm}$ & $0.49 \mathrm{~mm}$ & 0.345 \\
\hline 14 & M2- $\Delta y$ & $1 \mathrm{~mm}$ & $0.54 \mathrm{~mm}$ & 0.244 \\
\hline 15 & M2- $\Delta z$ & $0.5 \mathrm{~mm}$ & $0.94 \mathrm{~mm}$ & 0.141 \\
\hline 16 & M3- $\theta_{x}$ & $10 \mathrm{mrad}$ & $4.15 \mathrm{mrad}$ & 0.281 \\
\hline 17 & M3- $\theta_{u}$ & $10 \mathrm{mrad}$ & $3.11 \mathrm{mrad}$ & 0.281 \\
\hline 18 & M3- $\Delta z$ & $0.5 \mathrm{~mm}$ & $1.62 \mathrm{~mm}$ & 0.141 \\
\hline 19 & Stop- $\Delta x$ & $1 \mathrm{~mm}$ & $50 \mathrm{~mm}$ & 0.005 \\
\hline 20 & Stop- $\Delta y$ & $1 \mathrm{~mm}$ & $50 \mathrm{~mm}$ & 0.005 \\
\hline 21 & M4- $\theta_{x}$ & $10 \mathrm{mrad}$ & $3.16 \mathrm{mrad}$ & 0.100 \\
\hline 22 & M4- $\theta_{y}$ & $10 \mathrm{mrad}$ & $2.54 \mathrm{mrad}$ & 0.100 \\
\hline 23 & M4- $\Delta x$ & $1 \mathrm{~mm}$ & $0.23 \mathrm{~mm}$ & 0.398 \\
\hline 24 & M4- $\Delta y$ & $1 \mathrm{~mm}$ & $0.28 \mathrm{~mm}$ & 0.199 \\
\hline 25 & $\mathrm{M} 4-\Delta z$ & $0.5 \mathrm{~mm}$ & $0.87 \mathrm{~mm}$ & 0.199 \\
\hline 26 & $\operatorname{Im}-\theta_{x}$ & $50 \mathrm{mrad}$ & $16.8 \mathrm{mrad}$ & 0.031 \\
\hline 27 & $\operatorname{Im}-\theta_{y}$ & $50 \mathrm{mrad}$ & $14.1 \mathrm{mrad}$ & 0.031 \\
\hline 28 & $\operatorname{Im}-\Delta z$ & $2.5 \mathrm{~mm}$ & $3.89 \mathrm{~mm}$ & 0.063 \\
\hline 29 & M1-c & $10^{-7} \mathrm{~mm}^{-1}$ & $6.86 \mathrm{~mm}$ & 0.199 \\
\hline 30 & $\mathrm{M} 2-c$ & $10^{-7} \mathrm{~mm}^{-1}$ & $0.93 \mathrm{~mm}$ & 0.141 \\
\hline 31 & M3-c & $10^{-7} \mathrm{~mm}^{-1}$ & $0.60 \mathrm{~mm}$ & 0.199 \\
\hline 32 & M4-c & $10^{-7} \mathrm{~mm}^{-1}$ & $0.83 \mathrm{~mm}$ & 0.141 \\
\hline
\end{tabular}

Table 1. Elements $x_{j}$ of the configuration vector $\mathbf{x}$ used to describe the physical state of the EUV projection optics system. The shaded rows are the parameters selected as compensators. The capture ranges are found from the CodeV model of the optics, as described in Sec. 6 The initial configurations in the alignment simulations of Sec. [6 were generated from random distributions with $3 \sigma$ widths given by the product of the full-error capture range and the weighting factor. 


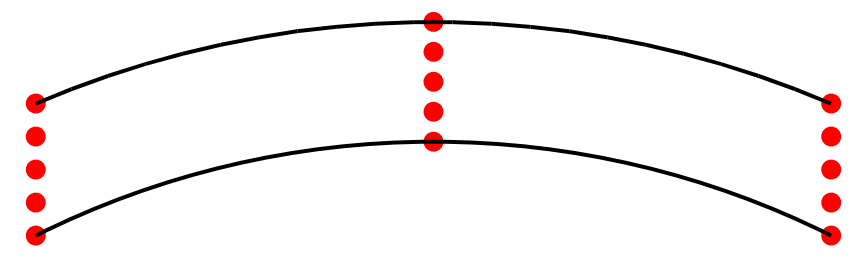

Figure 2. Schematic of the field points used in the definition of the aberration vector $\mathbf{z}$. The diagram is not to scale, and the field depicted has a chord length of $26 \mathrm{~mm}$ and a width of $1.5 \mathrm{~mm}$ at the image plane.

\section{ALIGNMENT AS A MINIMISATION OF ABERRATIONS}

\subsection{The aberration vector}

The optical performance of the system can be characterized by the aberration field, which is the field-dependent exit pupil wavefront error. The aberration vector is formed by decomposing the wavefront error into a sum of Zernike polynomial $\$ 11$ at a number of predetermined field points. The aberration vector, $\mathbf{z}$, is the long list of all the Zernike coefficients determined at all of these field points. The dimension of $\mathbf{z}, n_{z}$, is given by the product of the number of field points with the number of Zernike coefficients at each field point. The Zernike expansion includes the tilt terms but not the piston term (i.e. the mean wavefront is discarded from the expansion). The coefficients of the tilt terms are proportional to the distortion at the image plane, $\frac{12}{12}$ and in an experimental determination these terms may need to be measured by some method other than interferometry. It has been determined that the 15 field points shown schematically in Fig. 2 are sufficient to sample the dependence of all the aberration terms on field position.13. These field points are the most sensitive locations to measure all aberrations that can be addressed by the rigid-body motions of the optical elements. That is, in the absence of figure errors, there is no configuration that would result in aberrations that could degrade the imaging quality at any point in the field and yet not be detected at these field points.

Images in the EUV projection camera will be formed by synchronously scanning the mask and wafer, so the quality of a given printed point is the integrated effect of the aberrations along the scan path of that point. The illumination intensity may vary along that path, and it has been shown that the printed image quality may be estimated by weighting the Zernike coefficients depending on their location along a scan line $\frac{13}{13}$

In the case where all field weightings are unity the norm of the aberration function, $\|\mathbf{z}\|$, is given by $\left(z_{1}^{2}+z_{2}^{2}+\right.$ $\left.\cdots+z_{n_{z}}^{2}\right)^{1 / 2}$. Since the $z_{i}$ are coefficients of orthonormal polynomials and the mean has been omitted, the sum of the squares of the Zernike coefficients for any one field point is equal to $\sigma^{2}$, the variance of the wavefront error. Thus, we have

$$
\|\mathbf{z}\|=\sqrt{\sigma_{1}^{2}+\sigma_{2}^{2}+\cdots+\sigma_{n_{f}}^{2}},
$$

where $\sigma_{i}$ is the RMS wavefront error of the $i$ th field point and $n_{f}$ is the number of field points. For the EUV system, the 15 field points of Fig. 2 are arranged along three scan paths - one passing through the center of the field and the other two at the edges. Field weightings are chosen so that $\|\mathbf{z}\|$ is equal to the quadrature sum of the effective RMS wavefront errors of the three scanned image points 13 The aberration vector of the design configuration $\mathbf{x}_{o}$ has a norm $\left\|\mathbf{z}\left(\mathbf{x}_{o}\right)\right\|=0.039 \lambda$, where $\lambda$ is the EUV wavelength of $13.4 \mathrm{~nm}$. This corresponds to an average effective RMS error per printed point of $0.022 \lambda$. This value includes the effects of distortion on the scanned image.

\subsection{The sensitivity matrix}

The dependence of the $n_{z}$ functions on the $n_{x}$ parameters can be written succinctly in vector form as $\mathbf{z}=\mathbf{z}(\mathbf{x})$, and the best configuration optical performance is that for which $\|\mathbf{z}(\mathbf{x})\|$ is a minimum. Finding the configuration $\mathbf{x}$ which minimizes $\|\mathbf{z}(\mathbf{x})\|$ is the same problem encountered in optimizing optical designs, for which there are many different approaches. Considerations to take into account when deciding on a method to solve this problem are that the dependence of each element of $\mathbf{z}$ on $\mathbf{x}$ is non-linear, and there are interdependencies between the configuration parameters 
which makes the minimisation of $\|\mathbf{z}\|$ an ill-posed problem. The non-linearity requires an iterative procedure, which for the alignment problem should converge as rapidly as possible. A method that converges quadratically and can account for the interdependencies is Newton's method based on the singular value decomposition 14 (SVD), which is similar to the method of Grey! In Newton's method it is noted that $\|\mathbf{z}\|$ can be minimized by trying to find a solution to $\mathbf{z}(\mathbf{x})=0$. This system of equations is solved by making a Taylor expansion in the neighbourhood of a particular configuration. That is,

$$
\mathbf{z}(\mathbf{x}+\delta \mathbf{x})=\mathbf{z}(\mathbf{x})+\mathbf{J} \cdot \delta \mathbf{x}+O\left(\delta \mathbf{x}^{2}\right),
$$

where $\mathbf{J}$ is the $n_{z} \times n_{x}$ Jacobian matrix of the aberrations with $J_{i j}=\partial z_{i} / \partial x_{j}$. The matrix $\mathbf{J}$, especially when evaluated about the design configuration, is also known as the sensitivity matrix. We seek the correction to the configuration, $\delta \mathbf{x}$, that will set the aberrations to zero, $\mathbf{z}(\mathbf{x}+\delta \mathbf{x})=0$, in the case where we neglect all the non-linear terms. From Eqn. (2) the correction is the solution to

$$
\mathbf{J} \cdot \delta \mathbf{x}=-\mathbf{z}(\mathbf{x}) .
$$

The solution to Eqn. (3) extrapolates along the gradient of the aberration function to the plane $\mathbf{z}=0$. Due to the residual non-linearity of the aberrations the corrected aberration at $\mathbf{x}+\delta \mathbf{x}$ will not necessarily be minimized and so the process must be iterated.

For the purpose of aligning an optical system the Jacobian matrix can either be determined experimentally (requiring at least $n_{f} \times n_{\text {comp }}$ measurements, where $n_{\text {comp }}$ is the number of compensators) or from a model of the system (using the Jacobian evaluated at the design configuration: $\mathbf{J}_{o}=\mathbf{J}\left(\mathbf{x}_{o}\right)$, where $\mathbf{x}_{o}$ is the design configuration). For the purpose of characterising the system for compensator selection, the Jacobian is computed for all $n_{x}$ degrees of freedom, using the ray-tracing program CodeV 15

\subsection{The Singular Value Decomposition of the Sensitivity Matrix}

The SVD of the sensitivity matrix $\mathbf{J}_{o}$ is given by

$$
\mathbf{J}_{o}=\mathbf{U} \mathbf{W} \mathbf{V}^{T},
$$

where $\mathbf{U}$ and $\mathbf{V}$ are column-orthonormal matrices whose columns are the aberration singular vectors and the configuration singular vectors, respectively, and $\mathbf{W}$ is a diagonal matrix containing the corresponding singular values. The singular vectors are ordered so that their singular values are decreasing: $w_{1} \geq w_{2} \geq \cdots \geq w_{n_{x}} \geq 0$, where $w_{i}$ is the $i$ th singular value. The $i$ th configuration singular vector, $\mathbf{v}_{i}$, given by the $i$ th column of $\mathbf{V}$, is mapped to the $i$ th aberration singular vector since, from Eqn. (4) and the fact that $\mathbf{V}$ is orthonormal, $\mathbf{J}_{o} \mathbf{v}_{i}=w_{i} \mathbf{u}_{i}$. Each configuration singular vector has unit norm, as does each aberration singular vector, and hence the singular values have the same units as the Jacobian itself, viz. waves of aberration per unit adjustment. The singular values can be thought of as the sensitivity of the configuration singular vectors. A plot of the singular values of the EUV optics is given in Fig. 3, and some of the singular vectors are shown in Fig. [4.

The first singular vector, $\mathbf{v}_{1}$ shown in Fig. 4 is the most sensitive and has a singular value of 520 waves per unit adjustment. It is seen that this aberration is primarily defocus, achieved mainly by moving the image plane along the $z$-axis. The second largest independent aberration that can be achieved with an adjustment of unit magnitude is caused by a rotation of the object plane about its $y$-axis. This places the extremes of the used ring field out of focus and induces a large amount of distortion at those field points. The dominant aberration modes that the system will display when misaligned are given by the most sensitive singular vectors. The first four, shown in the figure, are a good representative set of these. They are primarily focus modes (curvature of field), tilt modes (distortion) and astigmatism modes. The significance of singular vectors of low sensitivity is that they indicate dependencies between the configuration vectors. For example, consider the configuration singular vector $\mathbf{v}_{24}$, shown in Fig. 固, This vector has a singular value of only 0.008 waves per unit adjustment. By inspecting this singular vector we see that an error in M4- $\Delta z$ (element 25), for example, could be compensated for by mainly adjusting M2- $\Delta z$ (element 15 ) and M3- $\Delta z$ (element 18). The compensation would leave the system in a configuration proportional to $\mathbf{v}_{24}$, but the effect of this singular vector on the aberration field is proportional to its singular value.

In aligning the optical system, we only need correct the orthogonal aberrations whose singular values are greater than some sensitivity, $w_{\text {lim }}$. The magnitude of any remaining uncorrected aberration will be always less than $w_{\text {lim }}\|\mathbf{x}\|$. 


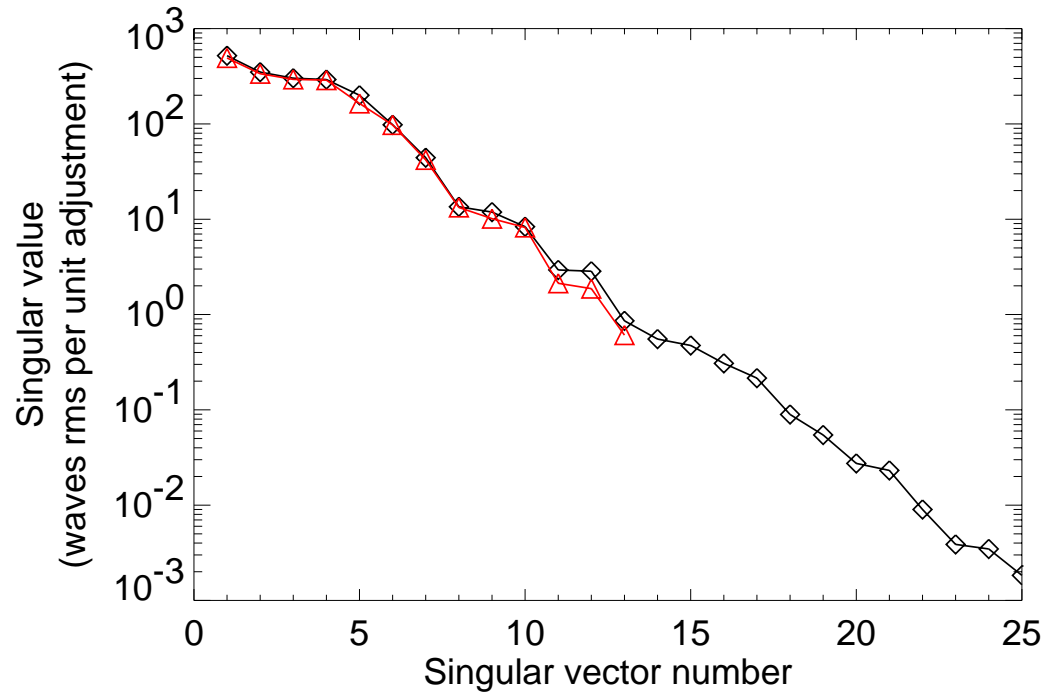

Figure 3. The singular values of the sensitivity matrix $\mathbf{J}_{O}$ (diamonds) and the singular values of the sub-matrix $\mathbf{J}_{\text {comp }}$, with $n_{\text {comp }}=13$ (triangles).

The magnitude of the initial misalignment and the required aligned RMS aberration determines the smallest sensitivity of a configuration singular value that must be corrected, which from Fig. 3 gives the number of orthogonal vectors that must be corrected. The set of singular vectors whose singular values are greater than $w_{\lim }$ form a subspace of configurations, which we refer to as the sensitive subspace. The dimension of this subspace, given by $n_{\text {sens }}$, is usually much less than the number of degrees of freedom of the system.

The corrections are simply found by decomposing the measured aberration field $\mathbf{z}$ into a sum of the aberration singular vectors, given by $\mathbf{U}^{T} \mathbf{z}$. If an aberration singular vector is in the sensitive subspace it is corrected by changing the configuration by an amount proportional to the corresponding configuration singular vector. The magnitude of the correction is the magnitude of the aberration divided by its sensitivity. The configurations in the "insensitive" subspace will not be corrected, but the effects of these misalignments will be small enough not to matter. This correction operation is expressed by the pseudo-inverse of $\mathbf{J}_{o}$, given by

$$
\delta \mathbf{x}=-\mathbf{V}_{\text {sens }} \frac{1}{\mathbf{W}_{\text {sens }}} \mathbf{U}_{\text {sens }}^{T} \mathbf{z}(\mathbf{x}),
$$

where the subscript "sens" refers to truncated matrices formed only from singular vectors in the sensitive subspace. The solution given by Eqn. (5) is regularized in that by ignoring the corrections to the insensitive aberrations it provides a correction of minimum norm.

\section{COMPENSATOR SELECTION}

The configuration singular vectors are not simple adjustments consisting of only one configuration parameter. Instead each singular vector may involve all possible parameters. That is, to make the correction of an aberration using Eqn (5D) would require making an adjustment on all the $n_{x}$ parameters, even though the correction is composed of $n_{\text {sens }}$ independent vectors. However, due to the redundancy of parameters, it is not necessary to use all parameters and it should be possible to make the correction by only utilising $n_{\text {sens }}$ parameters. The best set of compensators is that whose aberrations form the most linearly independent set within the sensitive subspace of aberrations. This mathematical problem appears often in a wide range of contexts, and is known as subset selection! 14 There are many methods for subset selection, including linear regression, $\frac{16}{16}$ the rank-revealing QR (RRQR) factorisation, 17 and using the SVD!14 We have made use of the SVD method of Golub, 14 acknowledged as the standard method, and an algorithm that is equivalent to the RRQR factorisation of Chan. $\frac{17}{17}$ The latter algorithm is listed in this section as Algorithm 1. 


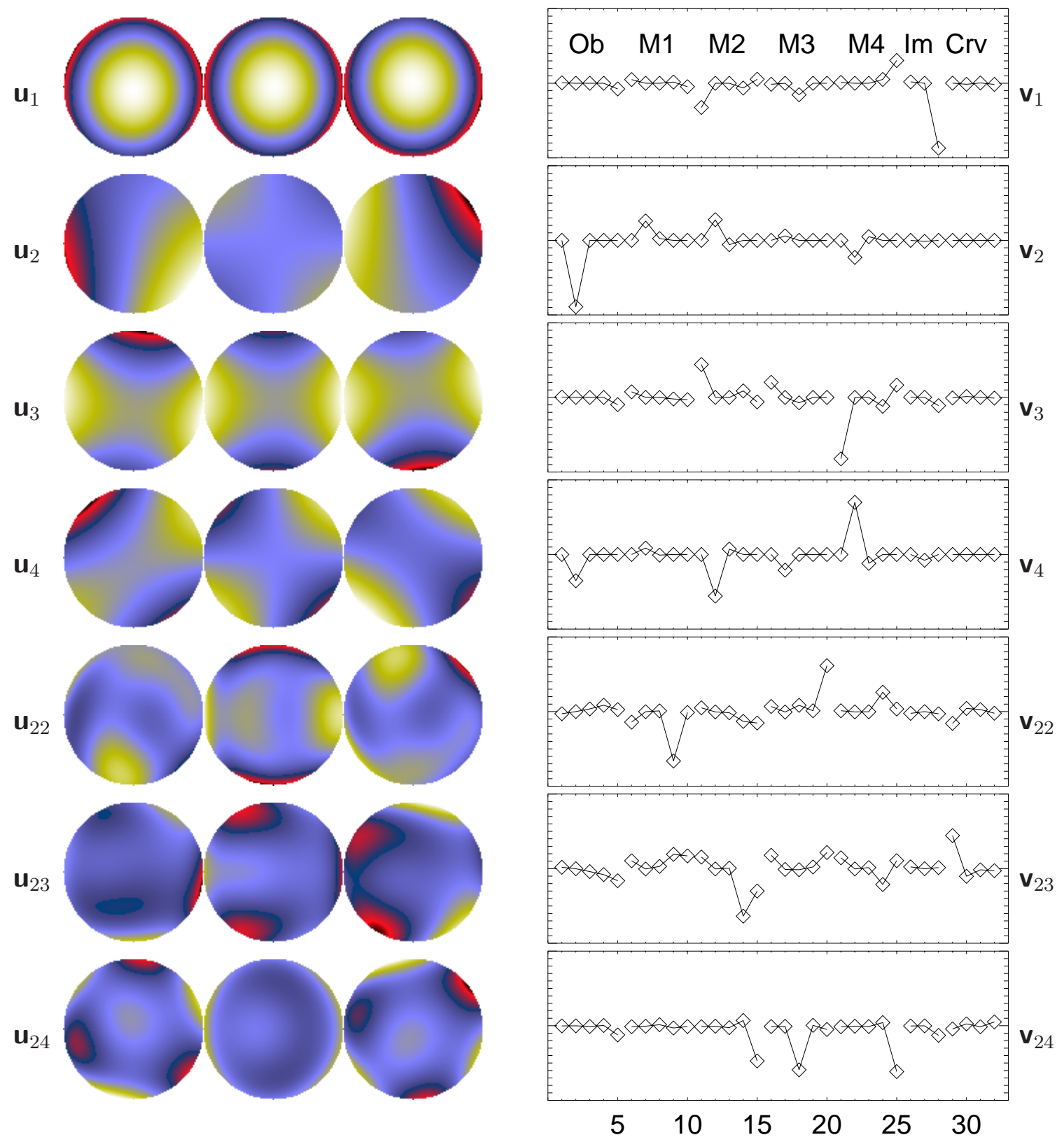

Figure 4. Selected singular vectors of the sensitivity matrix $\mathbf{J}_{o}$ for the EUV lithography system. The aberration vectors are shown as wavefront maps (including tilt) for field points at the ends and center of the ring field, and the configuration vectors are ordered as in Table 1 


\section{Algorithm 1 (Sequential subset selection)}

1. Form SVD of $\mathbf{J}_{o}: \mathbf{J}_{o}=\mathbf{U} \mathbf{W} \mathbf{V}^{T}$.

2. Initialise $\tilde{\mathbf{J}}=\mathbf{W} \mathbf{V}^{T}$, an $n_{x} \times n_{x}$ matrix.

3. For $i=n_{x}, n_{x}-1, \ldots n_{\mathrm{comp}}+1$ :

(a) Form the SVD of $\tilde{\mathbf{J}}$ and find the right singular vector $\mathbf{v}_{\min }$ of $\tilde{\mathbf{J}}$ with the smallest singular value.

(b) Find the element $j$ of $\mathbf{v}_{\min }$ with the largest absolute value.

(c) Remove column $j$ from $\tilde{\mathbf{J}}$ by setting it to zero.

4. The remaining $n_{\text {comp }}$ columns of $\tilde{\mathbf{J}}$ are the compensators.

Algorithm 1 is described as it applies to selection of compensators. As with the RRQR method we iterate and sequentially find parameters (columns of $\mathbf{J}_{o}$ ) that are least needed. Starting from the SVD of $\mathbf{J}_{o}$, given by Eqn. (4)), the configuration singular vector is found with the smallest singular value (this is the last singular vector, $\mathbf{v}_{n_{x}}$ ). The element of $\mathbf{v}_{n_{x}}$ with the largest absolute value is the parameter that we can most easily do without, since this is the parameter that is most effectively corrected for (using the complementary part of $\mathbf{v}_{n_{x}}$ ). This parameter is eliminated by setting its column of $\mathbf{J}_{o}$ to zero. The procedure for eliminating one parameter from the full matrix can be carried out on the sub-matrix and iterated until we have $n_{\text {sens }}$ parameters left or until we have reached the desired sensitivity. We refer to the sub-matrix of the Jacobian, consisting of columns corresponding only to the compensators, as $\mathbf{J}_{\text {comp }}$.

Performing this method with $n_{\text {comp }}=13$ we obtain the compensators that are highlighted as the grey rows in Table 1. Seven of the 13 compensators are motions of the mirrors and the remaining six are object and image plane motions. The mirror motions consist of $\theta_{x}$ and $\theta_{y}$ tilts of mirrors M2, M3, and M4, and the $\Delta z$ translation of mirror $\mathrm{M} 4$, and the object and image plane motions consist each of the two tilts and $\Delta z$ translation. The singular values of the matrix $\mathbf{J}_{\text {comp }}$, formed from these 13 compensators is compared with the singular values of $\mathbf{J}_{o}$ in Fig. 3. The close agreement between the singular values indicates that $\mathbf{J}_{\text {comp }}$ is addressing almost the same subspace as the sensitive subspace. In addition to the 13 compensators, we also include M2- $\Delta z$, the $x$ and $y$ translations of the stop, and the $x$ and $y$ location of the ring-field in the object plane. These extra adjustments allow better control of telecentricity and give a larger tolerance on the base-sphere curvature of the mirror surfaces.

\section{THE CAPTURE RANGE OF MISALIGNMENTS}

The ability to make corrections can be expressed by the capture range - the largest misalignment that can be corrected such that the residual aberration is within the alignment tolerance. The residual aberration that remains when making the correction to the aberration field $\mathbf{z}=\mathbf{J}_{o} \mathbf{x}$ by using only the compensators is given by $\mathbf{r}_{\text {comp }}=\mathbf{z}-\mathbf{J}_{o} \delta \mathbf{x}_{\text {comp }}$. The correction is given by $\delta \mathbf{x}_{\text {comp }}=\mathbf{J}_{\text {comp }}^{\dagger} \mathbf{z}$, where $\mathbf{J}^{\dagger}=\left(\mathbf{J}^{T} \mathbf{J}\right)^{-1} \mathbf{J}^{T}$ is the normal equations inverse of $\mathbf{J}$. The normal equations inverse can be used here since $\mathbf{J}_{\text {comp }}$ is much better conditioned than $\mathbf{J}_{o}$ (it is equivalent to the SVD pseudo-inverse when all singular vectors are included). Thus,

$$
\left\|\mathbf{r}_{\text {comp }}\right\| \leq\left\|\left(\mathbf{I}-\mathbf{J}_{\text {comp }} \mathbf{J}_{\text {comp }}^{\dagger}\right) \mathbf{J}_{o}\right\|\|\mathbf{x}\|=\omega_{\text {comp }}\|\mathbf{x}\|,
$$

where $\mathbf{I}$ is the $n_{\text {comp }} \times n_{x}$ identity matrix. The norm of the matrix expression in Eqn. (6) is given by its largest singular value ${ }^{14}$ denoted here by $\omega_{\text {comp }}$, and which can be computed quite quickly by SVD.

Equation (6) gives an upper bound on how large the residual error can possibly be after correcting a configuration whose magnitude of misalignment is $\|\mathbf{x}\|$. This upper bound represents the case where all of the uncorrected aberration is the most sensitive that it can be, i.e. it is given by the most sensitive singular vector that can not be corrected. A better estimate of the capture range can be found by considering a random configuration $\mathbf{x}_{r}$ for which the elements are unbiased and uncorrelated. The magnitude of $\mathbf{x}_{r}$ is distributed uniformly throughout its elements. Since the singular vectors are orthogonal, the magnitude of $\mathbf{x}_{r}$ will be distributed uniformly throughout the singular vectors, so that the expected value of the magnitude of each configuration singular vector in $\mathbf{x}_{r}$ is $E\left(\left|\mathbf{v}_{i}^{T} \mathbf{x}_{r}\right|\right)=\left\|\mathbf{x}_{r}\right\| / \sqrt{n_{x}}$, where $n_{x}$ is the dimension of the configuration vector. We then find that the RMS error of the residual aberration is

$$
\left\|\mathbf{r}_{\text {comp }}\right\| \approx \frac{1}{\sqrt{n_{x}}} \omega_{\text {comp }}\left\|\mathbf{x}_{r}\right\| .
$$




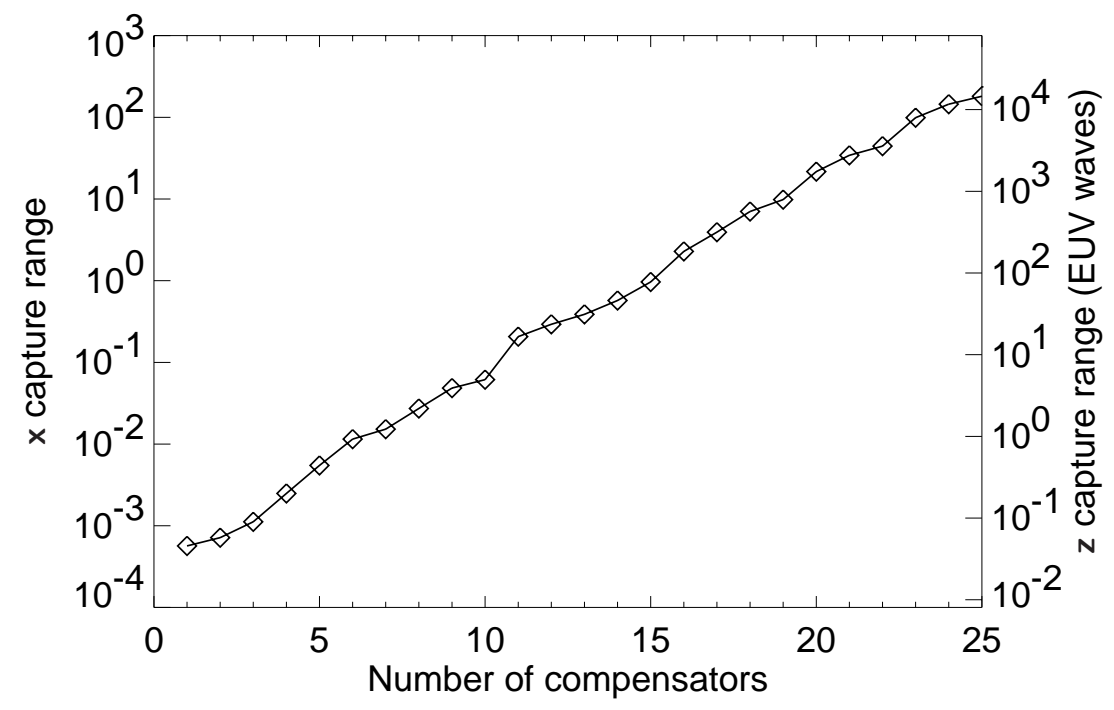

Figure 5. The approximate capture range for misalignment correction as a function of the number of compensators used. The left axis shows the capture range in terms of the size of the misalignment, $\|\mathbf{x}\|$, in the units specified in Table1, while the right axis shows it in terms of the magnitude of the uncorrected aberration.

If $\epsilon_{\text {align }}$ is the tolerance on the aberration of the aligned system, then the capture range is

$$
\left\|\mathbf{x}_{r}\right\| \approx \frac{\epsilon_{\mathrm{align}} \sqrt{n_{x}}}{\omega_{\mathrm{comp}}} .
$$

The capture range is plotted in Fig. [5 as a function of the number of compensators that are allowed in the alignment. The capture range was calculated for each number of compensators by first determining the best set of compensators using subset selection. Next the maximum sensitivity of the residual, $\omega_{\text {comp }}$, was computed from the definition in Eqn. (6). Finally, the capture range, as defined by Eqn. (8), was calculated. It is seen from Fig. 5 that each time an extra compensator is added the capture range increases by a factor of 1.8, on average. The wavefront error that can be tolerated after alignment was taken to be $\epsilon_{\text {align }}=\lambda / 35 \sqrt{3}$ ( $\lambda / 35$ for each scanned image).

It can now be seen from Fig. 5 that using 13 compensators will align a system that has a magnitude of misalignment of $\|\mathbf{x}\| \leq 0.3$ units of adjustment by mechanical means (the definition of units of adjustment is given in Table 1). If this error was uniformly distributed throughout all the configuration parameters, this would correspond to locating the optical surface to within $53 \mu \mathrm{m}$ and orienting it to within $0.53 \mathrm{mrad}$. The capture range achieved by using the 13 compensator motions can be compared with that achieved by using the 13 most sensitive singular vectors. It is found that for $n_{\text {comp }}=13$ the capture range of $\mathbf{J}_{\text {comp }}$ is 0.86 times that of $\mathbf{J}_{\text {sens }}$, which shows that this choice of compensators well spans the sensitive subspace. This point is amplified by comparing the singular value decompositions of $\mathbf{J}_{\text {comp }}$ and $\mathbf{J}_{o}$, shown in Fig. 3. The fact that the singular values of each are almost identical indicates that they are addressing almost the same subspace.

\section{ALIGNMENT SIMULATIONS}

The results of the previous sections analysing the EUV lithography camera were based solely on the approximation that the aberration field depends linearly on the change in configuration. To validate this analysis and to obtain a more accurate characterisation of the system we perform simulated alignments of the optical system, using the ray-tracing program $\mathrm{CodeV}$

The progress of an alignment of a random configuration is shown in Fig. 6. The alignment simulation was carried out in one case with no measurement noise and another with an aberration measurement noise corresponding to $0.02 \lambda$ RMS wavefront error and $10 \mathrm{~nm}$ RMS distortion error at each field point. In both cases the figure of the 

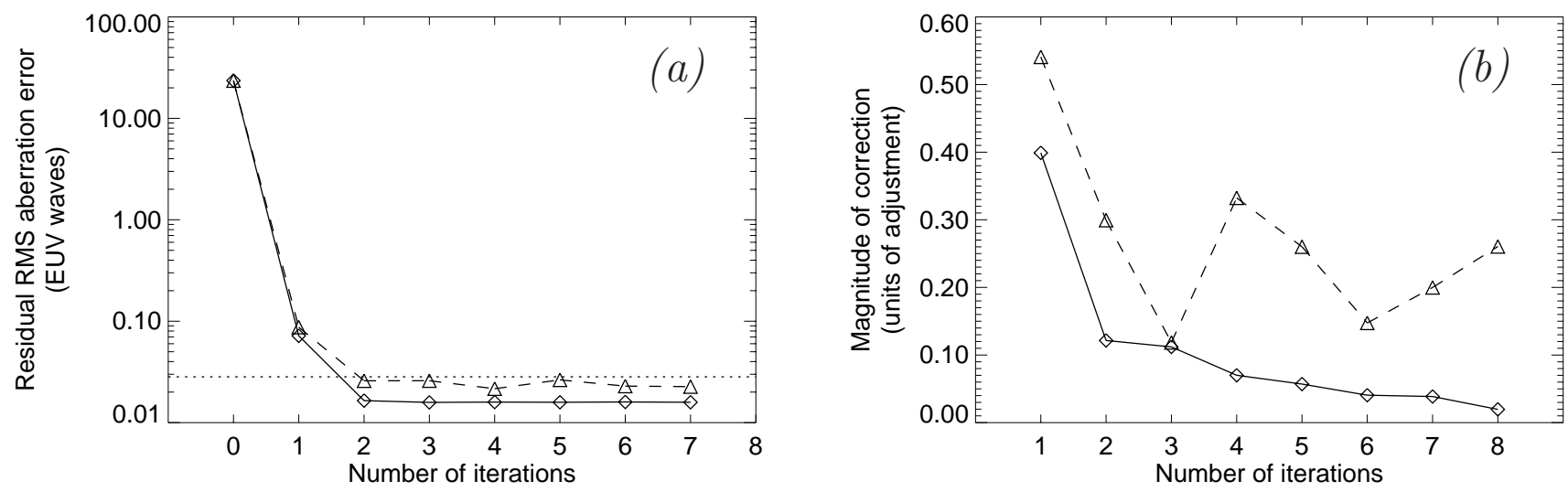

Figure 6. The residual RMS aberration error per scanned image (a), and the norm of the correction vector, $\|\delta \mathbf{x}\|$, (b) made at each iteration of the alignment procedure. The aberration error is the combined error of the tilt and wavefront, per scanned field, i.e. $\|\mathbf{z}\| / \sqrt{3}$. The simulation results with no measurement or figure error are plotted with diamonds, and the results with $\lambda / 50$ RMS error in wavefront determination and $10 \mathrm{~nm}$ RMS error in distortion determination are plotted with triangles. The alignment goal, $\epsilon_{\text {align }}=\lambda / 35$, is indicated.

optics was taken to be exactly as it was for the calculation of the Jacobian. That is, there was no error in the Jacobian. The initial configuration had a magnitude of 1.02 units of adjustment, and the initial aberration was 40.7 EUV waves (23.5 waves per printed field point). In both simulations the system was brought into specification after the second correction, and further corrections did not improve the aberration field significantly. When there was no measurement noise the final RMS aberration error was $\|\mathbf{z}\| / \sqrt{3}=0.017 \lambda$, as compared with $0.022 \lambda$ for the design. The improvement is primarily in the distortion. The decentered solution found by alignment is optimized for the particular scan weightings used here, which predict the reduction in Strehl ratio of the printed image due to the distortion-induced blurring! 13 Figure 6 shows that with noisy data, when the alignment gets to the point where the aberration to be corrected is of the same order of magnitude as the measurement error, the magnitude of the correction can be quite large. This implies that at the later iteration steps the alignment requires more regularisation, which can be achieved by eliminating the least sensitive singular vectors from $\mathbf{J}_{\text {comp }}$. This regularisation can be estimated by plotting the singular vector components of $\mathbf{z}$ and finding where they level off due to noise $\frac{18}{18}$

To test the validity of the capture range calculation shown in Fig. 5 , capture ranges were determined directly from the CodeV model by finding how far out of alignment each configuration parameter can be such that successful iterative alignment can still be achieved. This was carried out for each parameter in turn, by successively increasing the value of that parameter until the alignment was no longer successful. The results are given in Table 1. In this table the capture range is defined for misalignment of that parameter alone, given that all others are at the design configuration. Thus the capture range of the system is found from a weighted sum of the full-error capture ranges, such that the squares of the weights sum to unity. Choosing equal weights $\left(1 / \sqrt{n_{x}}\right)$ gives capture ranges of the same order of magnitude as predicted in Fig. 5. Unequal weightings were used for the simulations described, as listed in Table 1.

Alignments were carried out on a large number of random misalignments, and it was found that alignment was successful in all of the 200 simulations. Simulations were also carried out with initial misalignments at 1.5 and 3 times the capture range. In these cases the alignment was successful in $91 \%$ and $49 \%$ of the simulations. These results confirm that the capture ranges of Table 1 are indeed accurate, and the probability of aligning the system decreases as the misalignment increases beyond the capture range.

In another study alignments were carried out with different amounts of measurement noise and error in the figure of the surfaces. Figure 7 shows the magnitude of the residual aberration for various degrees of noise. For each data point 50 alignment simulations were carried out, each one having a different random initial configuration and different random noise. The initial configuration was always generated from distributions with $3 \sigma$ widths given by the capture ranges and weightings in Table 1. The plot of the residual aberration versus measurement noise in Fig. 7 (a) shows that the residual error is approximately equal to the quadrature sum of the measurement noise and 

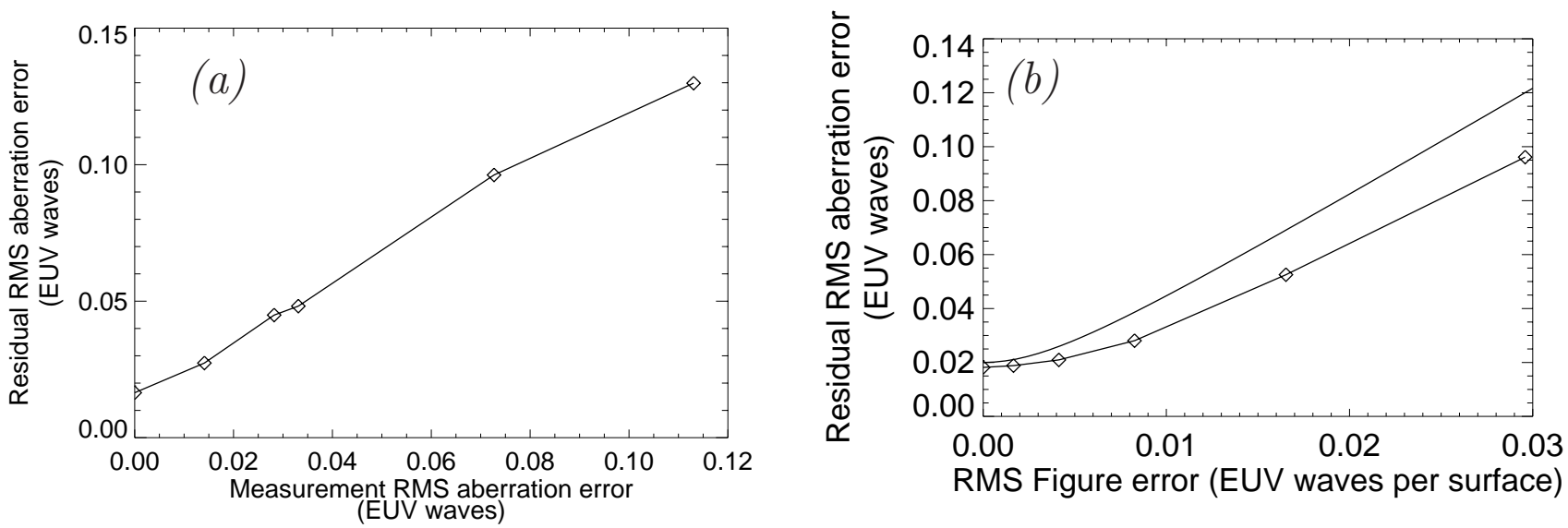

Figure 7. (a) The residual RMS aberration error achieved for different amounts of noise in the measurement of wavefront and distortion. The actual RMS wavefront errors used were (in order of increasing RMS aberration error) $0.005 \lambda, 0.01 \lambda, 0.02 \lambda, 0.05 \lambda$, and $0.1 \lambda$ and the RMS distortion errors were $5 \mathrm{~nm}, 10 \mathrm{~nm}, 10 \mathrm{~nm}, 20 \mathrm{~nm}$, and $20 \mathrm{~nm}$. (b) The residual RMS aberration error obtained with various amounts of RMS error in the figure of the four optical surfaces. The measurement noise in this case was zero.

the aberration error of the noise-free alignment. The dependence of the residual RMS aberration error on figure error is plotted in Fig. 7 (b). It is found that the alignment actually compensates for a small amount of figure error. At the design configuration $\mathbf{x}_{o}$, the addition of an RMS error of $\sigma_{\text {fig }}$ to each surface gives a wavefront error of $4 \sigma_{\text {fig }}$ (each uncorrelated surface contributes a wavefront error of $2 \sigma_{\text {fig }}$ ).

\section{CONCLUSIONS}

A method was presented on how to choose the best set of compensators to align a precision optical system. The selection is based on an analysis of the sensitivity matrix of the system, which describes to first order the way the aberrations change for a given change in the configuration of the optical elements. The selected compensators correspond to the sub-matrix of the sensitivity matrix which best addresses the sensitive orthogonal aberrations. The alignment of the system, using these compensators, corrects only the sensitive orthogonal aberrations. The remaining orthogonal aberrations will not be corrected, but their sensitivity is too low to have any effect on the system.

The analysis yields some fundamental characteristics about the imaging system, including how many compensators are required to correct a misalignment of a given magnitude, the dominant aberrations induced by changes in the configuration, and the dependencies between configuration parameters. For example, for the EUV projection optics, it is found that each time an optimally-chosen compensator is added the magnitude of the misalignment that can be corrected is increased on average by a factor of 1.8. With 13 compensators, eight of which are adjustments of the rigid-body motions of the optics, misalignments yielding about 30 waves of aberration per scanned image point can always be reduced to $\lambda / 50$ when there is no measurement noise.

Simulations of the alignment of the EUV projection optics show that the procedure is robust to measurement noise and errors in the sensitivity matrix due to imperfect knowledge of the surface figure. The simulations show that to meet the Maréchal criterion (which is still not good enough for production instruments $)^{11}$ requires wavefront measurements to be made to $0.2 \mathrm{~nm}$ RMS, distortion measurements to $10 \mathrm{~nm}$ RMS, and optics to be figured to $0.25 \mathrm{~nm}$ RMS. In this case the image quality of the scanned image will meet the criterion. That is, the distortioninduced blurring is accounted for. Technology improvements are being made at a rapid rate and will provide EUV projection optics of this and even higher quality.

\section{ACKNOWLEDGEMENTS}

This work was performed under the auspices of the U.S. Department of Energy by the Lawrence Livermore National Laboratory under Contract No. W-7405-ENG-48. Funding was provided by the Extreme Ultraviolet Limited Liability Corporation under a Cooperative Research and Development Agreement. 


\section{REFERENCES}

1. D. M. Williamson, "The elusive diffraction limit," in OSA Proceedings on Extreme Ultraviolet Lithography, F. Zernike and D. T. Attwood, eds., vol. 23, pp. 68-76, Optical Society of America, (Washington, DC), 1995.

2. J. S. Taylor, G. E. Sommargren, and D. W. Sweeney, "Fabrication and testing of optics for EUV projection lithography," in Emerging Lithographic Technologies II, Proc. SPIE 3331, 1998.

3. G. E. Sommargren, "Diffraction methods raise interferometer accuracy," Laser Focus World 32, pp. 61-71, August 1996.

4. P. Naulleau, K. A. Goldberg, E. Tejnil, S. H. Lee, H. Medecki, C. J. Bresloff, P. J. Batson, P. Denham, D. T. Attwood, Jr., and J. Bokor, "Characterization of the accuracy of EUV point diffraction interferometry," in Emerging Lithographic Technologies II, Proc. SPIE 3331, 1998.

5. H. W. Jeong, G. N. Lawrence, and K. B. Nahm, "Auto-alignment of a three-mirror off-axis telescope by reverse optimisation and end-to-end aberration measurements," in Current developments in optical engineering II, Proc. SPIE 818, pp. 419-430, 1987.

6. T. Haga and M. C. K. Tinone, "Assembly of extreme ultraviolet lithography optics using at-wavelength Foucault testing," Jpn. J. Appl. Phys. 35, pp. 6480-6486, 1996.

7. D. M. Williamson, "Compensator selection in the tolerancing of a microlithographic lens," in Recent trends in optical systems design II, Proc. SPIE 1049, pp. 178-186, 1989.

8. W. H. Press, S. A. Teukolsky, W. T. Vetterling, and B. P. Flannery, Numerical Recipes in C: the art of scientific computing, Cambridge University Press, Cambridge, 2nd ed., 1992.

9. D. S. Grey, "Aberration theories for semi-automatic lens design," J. Opt. Soc. Am. 55, pp. 672-676, 1963.

10. D. W. Sweeney, R. M. Hudyma, H. N. Chapman, and D. R. Shafer, "EUV optical design for a $100 \mathrm{~nm}$ CD imaging systems," in Emerging lithographic technologies II, Proc. SPIE 3331, 1998.

11. V. N. Mahajan, "Zernike circle polynomials and optical aberrations of systems with circular pupils," Appl. Opt. 33, pp. 8121-8124, 1994.

12. V. N. Mahajan, Aberration theory made simple, SPIE Press, Washington, 1991.

13. H. N. Chapman and D. W. Sweeney, "Compensator selection and assembly of precision optical systems." in preparation.

14. G. H. Golub and C. F. Van Loan, Matrix Computations, Johns Hopkins University Press, Baltimore, 3rd ed., 1996.

15. Optical Research Associates, http://www.opticalres.com

16. A. J. Miller, Subset selection in regression, Chapman and Hall, New York, 1990.

17. T. F. Chan and P. C. Hansen, "Some applications of the rank revealing QR factorization," SIAM J. Sci. Stat. Comput. 13, pp. 727-741, 1992.

18. P. C. Hansen, "Regularization tools: A Matlab package for analysis and solution of discrete ill-posed problems," Num. Alg. 6, pp. 1-35, 1994. 


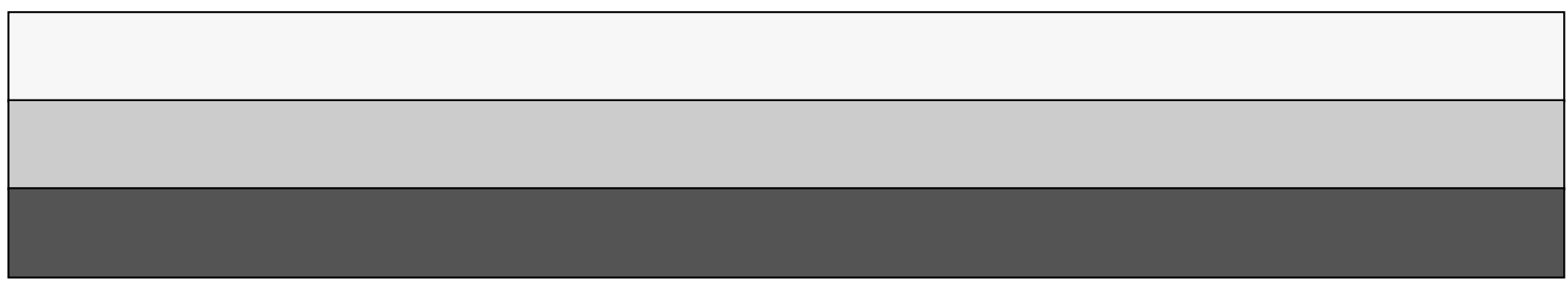

